\title{
Synthesis and characterization of membranes obtained by graft copolymerization of 2-hydroxyethyl methacrylate and acrylic acid onto chitosan
}

\author{
K.S.C.R. dos Santos ${ }^{a, b, *}$, J.F.J. Coelho ${ }^{b}$, P. Ferreira ${ }^{b}$, I. Pinto ${ }^{b}$, \\ S.G. Lorenzetti ${ }^{\mathrm{c}}$, E.I. Ferreira ${ }^{\mathrm{a}}$, O.Z. Higa ${ }^{\mathrm{c}}$, M.H. Gil ${ }^{\mathrm{b}}$ \\ ${ }^{a}$ Faculdade de Ciências Farmacêuticas, Universidade de São Paulo, Av. Prof. Lineu Prestes, 580, Bloco 13, 05508-900 São Paulo, Brazil \\ ${ }^{\mathrm{b}}$ Departamento de Engenharia Química, Faculdade de Ciência e Tecnologia, Universidade de Coimbra, \\ Polo II, Pinhal de Marrocos, 3030-290 Coimbra, Portugal \\ ${ }^{c}$ Instituto de Pesquisas Energéticas e Nucleares, IPEN/CNEN, Av. Prof. Lineu Prestes, 2242, 05508-900 São Paulo, Brazil
}

Received 26 July 2005; received in revised form 2 November 2005; accepted 3 November 2005

Available online 18 January 2006

\begin{abstract}
Chitosan based membranes to be applied on wound healing as topical drug delivery systems were developed by graft copolymerization of acrylic acid (AA) and 2-hydroxyethyl methacrylate (HEMA) onto chitosan using cerium ammonium nitrate as chemical initiator. Evidence for graft copolymerization of the vinyl monomers onto chitosan was obtained by FTIR and DMTA. Swelling degree, cytotoxicity, thrombogenicity and haemolytic activity of these membranes were evaluated. Chitosan-graft-AA-graft-HEMA showed to be the best matrix for drug delivery systems than chitosan-graft-AA because it retains good swelling properties, but the content in HEMA has improved cytocompatibility, hemocompatibility and thrombogenic character.
\end{abstract}

(c) 2005 Elsevier B.V. All rights reserved.

Keywords: Chitosan; Acrylic acid; 2-Hydroxyethyl methacrylate; Membranes; Wound healing

\section{Introduction}

In the past, wounds were treated by allowing them to dry and, hence, acquire a hard protective coating, the scab. About 30 years ago, the treatment of wounds was revolutionized when it was discovered that a wound would heal faster when a 'moist' dressing was applied rather than the traditional 'dry' dressings such as gauze type materials produced from cotton or lint. Natural skin is recognized as the ideal wound dressing and so the development of 'moist' dressings was based on the interest on developing substitutes of skin with its $85 \%$ water content and inherent permeability. In this context, wound management aids are available in a range of physical forms including fibres and threads, films for use as a covering and gels (Lloyd et al., 1998). Polysaccharides, as natural biomolecules, are obvious choice for investigation as potential wound substitutes. The polysaccha-

\footnotetext{
* Corresponding author. Tel.: +55 113091 3687; fax: +55 1138154418 E-mail address: katiasol@usp.br (K.S.C.R. dos Santos).
}

rides and its derivatives may, by themselves, actively participate in the process of wound healing. As examples, hyaluronic acid and chitosan can be used for this purpose.

Chitin, the second most abundant natural polysaccharide, found in the exoskeleton of crustaceous, the cuticles of insects, and the cell walls of fungi, is a linear homopolymer composed of $\beta(1-4)$-linked $N$-acetyl glucosamine (Li et al., 1997). Partial deacetylation of chitin results in the production of chitosan, which is a polysaccharide composed by copolymers of glucosamine and $N$-acetyl glucosamine (Singla and Chawla, 2001).

Chitosan is currently receiving a great deal of attention for medical and pharmaceutical applications. The main reasons for this are undoubtedly its appealing intrinsic properties, such as biocompatibility, biodegradability, non-toxicity, adsorption properties, film-forming ability, bioadhesivity, antimicrobial activity against fungi, bacteria and viruses, in addition to its haemostatic effect (Henriksen et al., 1996; Rao and Sharma, 1997; Illum, 1998; Ravi Kumar, 2000; Chirkov, 2002; Lim and Hudson, 2003; Okamoto et al., 2003; Rabea et al., 2003; Zheng and Zhu, 2003). Most of the properties of chitosan can be related 
to its cationic nature. At acidic $\mathrm{pH}$, it is a linear polyelectrolyte with a high charge density with one positive charge per glucosamine residue and, therefore, will interact with negatively charged molecules including proteins, anionic polysaccharides and nucleic acids (Olsen et al., 1988; Lloyd et al., 1998).

As Lloyd et al. (1998) have reported, in the area of wound healing, chitosan can reduce scar tissue (fibroplasia) by inhibiting the formation of fibrin in wounds. Chitosan can enhance blood coagulation by an independent mechanism of the classical coagulation cascade and appear to be an interaction between negative charges of cell membranes of erythrocytes and positive charges of chitosan filaments (Rao and Sharma, 1997; Okamoto et al., 2003). One hypothesis advanced to explain the ability of chitosan to enhance wound healing is related to its biodegradability (Berger et al., 2004). Lysozyme, normally produced by macrophages, hydrolyses chitosan and its derivatives to oligomers, which activate macrophages to produce nitric oxide, activated oxygen species, tumor necrosis factor- $\alpha$, interferon and interleukin-1. Activated macrophages increase the production of lysozyme, chitinase and $N$-acetyl- $\beta$-D-glucosaminidase, which catalyse the total depolymerization to monomers. The monomeric aminosugars become available to fibroblasts which proliferate under the action of interleukin-1, for incorporation into chondroitin 4- and 6-sulfate, hyaluronan and keratin sulfate, thus guiding the ordered deposition of collagen and also accelerating the wound healing process (Muzzarelli, 1991, 1997). All these properties, including its bioadhesivity, make chitosan an excellent biomaterial to treat wounds and scars.

Modifications on the chitosan structure can be carried out in order to adequate it to the intended application, such as hydrosolubility, adhesivity, haemocompatibility and others. The modification of polymeric materials by graft copolymerization has been studied deeply by several authors (Kurika, 1996; Harish Prashanth and Tharanathan, 2003; Sashiwa and Aiba, 2004) because it can provide materials with desired properties through the appropriate choice of the molecular characteristics of the side chain to be grafted (Casimiro et al., 2005). Chitosan bears two types of reactive groups that can be modified by grafting: the C-2 free amino groups on deacetylated units and the hydroxyl groups in the C-3 and C- 6 either in acetylated or deacetylated units (Lloyd et al., 1998; Berger et al., 2004) (Fig. 1). The reactive amino group in chitosan is important in several targetedstructural modifications since the deprotonated amino group acts as a powerful nucleophile $\left(\mathrm{p} K_{\mathrm{a}} \cong 6.3\right)$, readily reacting with electrophilic reagents (Chen et al., 2000).

The main objective of this study was to obtain membranes for wounds treatment with dual effects: to accelerate wound healing due to the bioactivities of chitosan itself and at the same time the polymeric matrix should act as a delivery system for many drugs to prevent or treat bacterial infections. With this purpose, new material was synthesized by grafting vinyl monomers, AA and HEMA, onto chitosan. The poly(AA), which is biocompatible and with antibacterial properties, is widely used in adhesives and super absorbents materials due to its pendant carboxylic groups (Lee et al., 1999, 2005; Athawale and Lele, 2001). Polymers grafted by AA become highly hydrophilic materials and interesting matrixes for drug delivery systems. They make a good wound dressing due to their content in water ('moist' dressing) and, once membranes can absorb large contents in water, they are able to retain more drug into their matrix when dried membranes are immersed on drug aqueous solution. Some of previous studies have shown that the presence of HEMA in copolymers improves the biocompatibility of these materials (Carenza, 1992; Singh and Ray, 1994).

\section{Material and methods}

\subsection{Material}

Chitosan, with a 0.15 acetylation degree (DA) and a 145,000 viscosimetric molecular weight was purchased from Aldrich Chemical Company Inc. DA was calculated from ${ }^{1} \mathrm{H}$ RMN in an ADPX $300 \mathrm{MHz}$ spectrometer from Brüker. The intrinsic viscosity was performed with an AVS-350 viscometer coupled to an AVS-50 automatic dilution module, both from Schott-Geräte. AA, HEMA and cerium ammonium nitrate were purchased from Aldrich Chemical Company Inc. Acetic acid, calcium chloride and formaldehyde were purchased from Merck \& Co. Inc. Phosphate buffered saline pH 7.4 (PBS), RPMI 1640 culture medium, fetal bovine serum (FBS) and trypsin were purchased from Gibco ${ }^{\mathrm{TM}}$ Cell Culture Products. The corants 3-(4,5-dimethylthiazol-2-yl)-5(3-carboxymethoxyphenyl)-2-(4-sulphophenyl-2H-tetrazolium (MTS) and phenazine methosulfate (PMS) were purchased from Promega Corporation.

\subsection{Graft copolymerization}

A $2 \%(\mathrm{w} / \mathrm{v})$ chitosan stock solution was prepared in $1 \%(\mathrm{v} / \mathrm{v})$ acetic acid aqueous solution. Fifteen milliliters of chitosan stock solution was stirred with $50 \mathrm{mg}$ of cerium ammonium nitrate for $10 \mathrm{~min}$. AA and HEMA monomers, which were used in different molar rates according to Table 1, were added to this solution and stirred until complete homogenization. The resulting solutions were transferred to Petri dishes and allowed to react for $24 \mathrm{~h}$ at $55^{\circ} \mathrm{C}$. Resultant films that formed were obtained and removed from the Petri dishes by adding $1 \%$ sodium hydroxide aqueous solution. They were extracted in a Soxhlet for a $6 \mathrm{~h}$ reflux using distilled water as a solvent to remove the homopolymer or nonreacted monomers. Finally, the membranes were dried at room temperature on Teflon ${ }^{\circledR}$ surfaces.

\subsection{Infrared analysis}

FTIR analysis of grafted chitosan films was performed in Magma-IR ${ }^{\mathrm{TM}}$ Spectrometer 750 from Nicolet Instrument Corp. equipped with Golden Gate Single Reflection Diamond ATR.

\subsection{Thermal properties}

Thick specimens $(15.20 \mathrm{~mm} \times 7.45 \mathrm{~mm} \times 1.10 \mathrm{~mm})$ were analysed by dynamical mechanical thermal analysis (DMTA). A Triton Tritec 2000 analyser was used in the constrain layer damping mode, with a standard heating rate of $10^{\circ} \mathrm{C} \mathrm{min}^{-1}$, at 

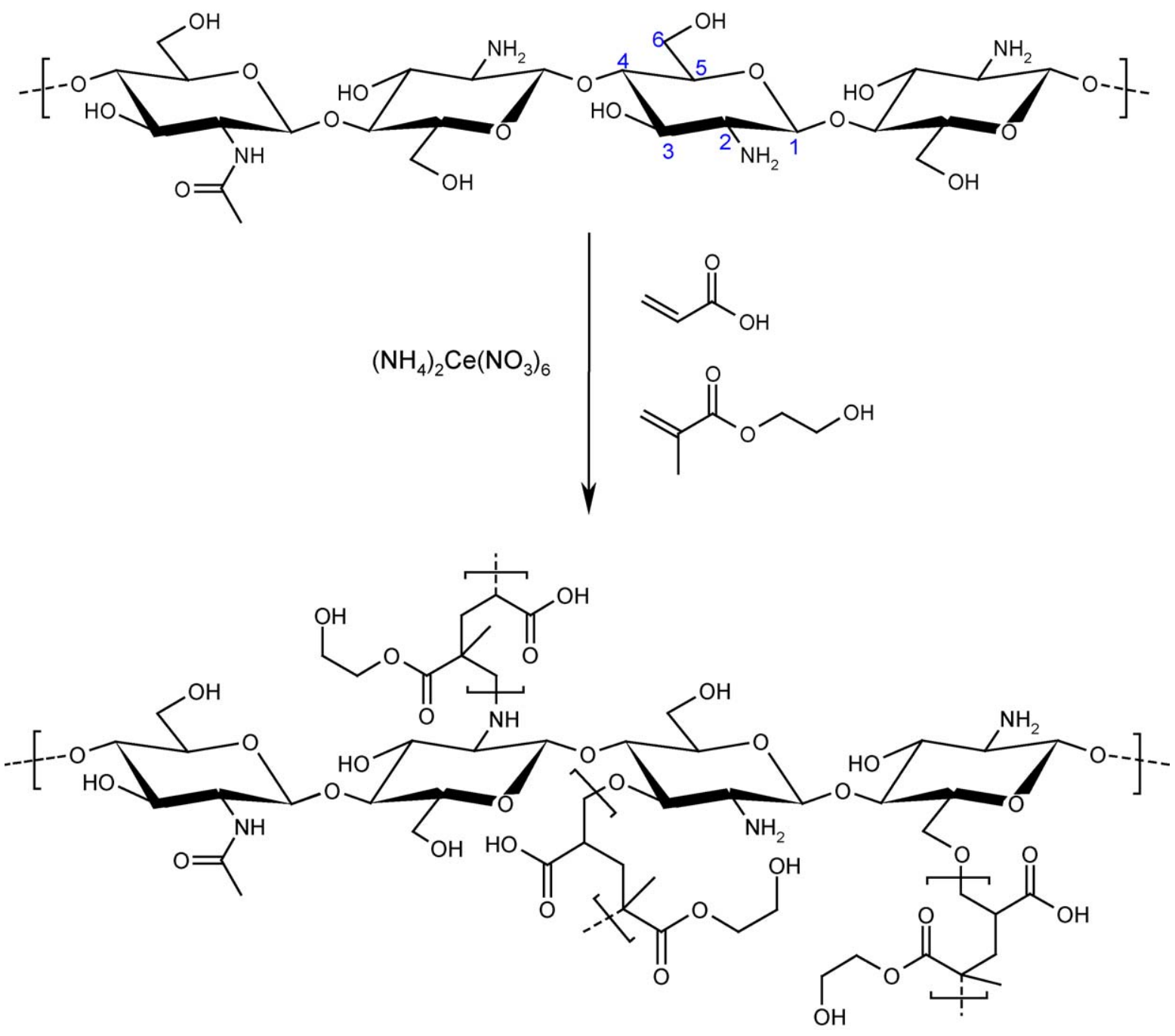

Fig. 1. Schematic representation of graft copolymerization of HEMA and AA onto chitosan.

frequency of $1 \mathrm{~Hz}$ and a displacement of $0.05 \mathrm{~mm}$. The glass transition temperature was determined as the peak in $\tan \delta$ $\left(\tan \delta=E^{\prime \prime} / E^{\prime}\right)$ where $E^{\prime \prime}$ and $E^{\prime}$ are the loss and storage modulus, respectively, derived from DMTA. All samples were dried for $24 \mathrm{~h}$ at $55^{\circ} \mathrm{C}$ before thermal analysis.

\subsection{Swelling studies}

Dried samples $\left(W_{\mathrm{d}}\right)$ were immersed in distilled water for $48 \mathrm{~h}$. The swollen samples $\left(W_{\mathrm{s}}\right)$ were removed from the solution, quickly wiped with filter paper and weighed. The maximum water uptake $(Q)$ of membranes was determined according to the following expression:

$Q=\left[\frac{W_{\mathrm{s}}-W_{\mathrm{d}}}{W_{\mathrm{d}}}\right] \cdot 100$

\subsection{Haemocompatibility}

The haemocompatibility was evaluated in vitro according to the International Standard Organization (ISO) (ISO 10993-4, 1999), and the following categories of blood

Table 1

Reactional conditions for synthesis of membranes

\begin{tabular}{|c|c|c|c|c|c|c|c|}
\hline \multirow[t]{2}{*}{ Sample } & \multicolumn{2}{|l|}{ chitosan } & \multirow[t]{2}{*}{$\left(\mathrm{NH}_{4}\right)_{2} \mathrm{Ce}\left(\mathrm{NO}_{3}\right)_{6}(\mathrm{mg})$} & \multicolumn{2}{|l|}{ HEMA } & \multicolumn{2}{|l|}{ AA } \\
\hline & $(\mathrm{mmol})$ & $(\mathrm{mL})^{\mathrm{a}}$ & & $(\mathrm{mmol})$ & $(\mathrm{mL})$ & $(\mathrm{mmol})$ & $(\mathrm{mL})$ \\
\hline M1 & 1.86 & 15 & - & - & - & - & - \\
\hline M2 & 1.86 & 15 & 50 & 12 & 2.92 & - & - \\
\hline M3 & 1.86 & 15 & 50 & - & - & 12 & 1.64 \\
\hline M4 & 1.86 & 15 & 50 & 6 & 1.46 & 6 & 0.82 \\
\hline
\end{tabular}

${ }^{a}$ A $2 \%(w / v)$ chitosan solution. 
interactions were studied: thrombogenicity and haemolytic potential.

\subsubsection{Thrombogenicity}

The evaluation of thrombus formation on polymeric surfaces was carried out using a gravimetric method (Imai and Nose, 1972). Anticoagulated rabbit blood was used with this purpose. This sample was prepared by adding $1 \mathrm{~mL}$ of anticoagulant acid citrate dextrose solution (ACD) (US Pharmacopeia XXIII, 1994) to $9 \mathrm{~mL}$ of fresh rabbit blood. Before performing the tests, the membranes were immersed in PBS at a constant temperature of $37^{\circ} \mathrm{C}$. After $48 \mathrm{~h}$ of incubation, the PBS was removed and the ACD blood was put in contact with the surface of the polymers and also to an empty Petri dish, which acted as a positive control. Blood clotting tests were initiated by adding $0.02 \mathrm{~mL}$ of a $10 \mathrm{M}$ calcium chloride solution and were stopped after $45 \mathrm{~min}$ by the addition of $5 \mathrm{~mL}$ of water. The resultant clots were fixed with $5 \mathrm{~mL}$ of a $36 \%$ formaldehyde solution and were then dried with tissue paper and finally weighed.

\subsubsection{Haemolytic potential}

The haemolysis tests were performed as described in American Society for Testing and Materials (ASTM) (ASTM F $756-00,2000)$. Samples $\left(21 \mathrm{~cm}^{2}\right)$ were placed in polypropylene test tubes and $7 \mathrm{~mL}$ of PBS were added. After $72 \mathrm{~h}$ of incubation at $37^{\circ} \mathrm{C}$, the PBS was removed and $1 \mathrm{~mL}$ of diluted ACD rabbit venous blood $(9.02 \mathrm{mg} / \mathrm{mL})$ was added to each sample and maintained at $37^{\circ} \mathrm{C}$ for $3 \mathrm{~h}$. Positive and negative controls were prepared by adding the same amount of ACD blood to $7 \mathrm{~mL}$ of water and PBS, respectively. Each tube was gently inverted twice each $30 \mathrm{~min}$ to maintain contact of the blood with the material. After incubation, each fluid was transferred to a suitable tube and centrifuged at $2000 \mathrm{rpm}$ for $15 \mathrm{~min}$. The haemoglobin released by haemolysis was measured by the optical densities (OD) of the supernatants at $540 \mathrm{~nm}$ using a spectrophotometer UV-vis (Jasco V-550). The percentage of haemolysis was calculated as follows

Haemolysis $(\%)=\left[\frac{(\text { OD sample }- \text { OD negative control })}{(\text { OD positive control }- \text { OD negative control })}\right]$

\subsubsection{Extract preparation}

According to the ISO 10993-5 (1992), the ratio between the surface area of the material and the volume of extraction vehicle should be in a range of 0.5 and $6.0 \mathrm{~cm}^{2} / \mathrm{mL}$. Hence, membrane samples of $4 \mathrm{~cm}^{2}$ were sterilized by autoclaving at $120^{\circ} \mathrm{C}$ for $20 \mathrm{~min}$ and then $4 \mathrm{~mL}$ of RPMI 1640 containing $10 \%$ FBS were added and stationary incubated at $37^{\circ} \mathrm{C}$ for 2 days. The extract obtained was employed for the cytotoxicity assay.

\subsubsection{Cytotoxicity assay}

Chinese hamster ovary $(\mathrm{CHO})$ cells were grown in culture medium composed of RPMI 1640 containing 10\% FBS, in a plastic tissue culture flask, at $37^{\circ} \mathrm{C}$ in a humidified $5 \% \mathrm{CO}_{2}$ air incubator. After confluent monolayer propagation, the culture medium was removed and the cells were washed with PBS. The culture was treated with $0.25 \%$ trypsin solution to detach cells from the culture tissue flask. After trypsinization, cells were transferred to a screw-capped plastic tube, centrifuged and washed twice with PBS. The cells were ressuspended in culture medium and the concentration was adjusted to $\sim 60,000$ cells $/ \mathrm{mL}$. Ninety six-well tissue culture microtiter plates were prepared by the addition of the biomaterial extract ( $50 \mu \mathrm{L} /$ well in quadruplicate), followed by the addition of $50 \mu \mathrm{L}$ of the cell suspension ( $\sim 3000$ cells). Blank was prepared with culture medium without cells and a negative control was prepared with culture medium instead of extract plus cells, both in quadruplicates. Extract solution of HDPE (high density poly(ethylene)) and $0.3 \%$ phenol solution were assayed such as negative and positive material controls, respectively. Microplates were incubated in a $5 \% \mathrm{CO}_{2}$ humidified atmosphere. After $72 \mathrm{~h}, 20 \mu \mathrm{L}$ of a mixture (20:1) of $0.2 \%$ MTS and $0.09 \%$ PMS in PBS were added to the test wells and left for $2 \mathrm{~h}$. Incorporated dye was measured by reading the absorbance at $490 \mathrm{~nm}$ in a microplate reader against the blank.
According to ASTM F 756-00 (2000) materials can be classified in three different categories according to their haemolytic index (haemolysis\%). Materials with percentages of haemolysis over $5 \%$ are considered haemolytic; while the ones with haemolytic index between $5 \%$ and $2 \%$ are classified as slightly haemolytic. Finally, when the material presents a haemolysis percentage below $2 \%$ it is considered as a non-haemolytic material.

\subsection{Cytotoxicity test}

The cytotoxicity assay of the biomaterial was carried out according to ISO 10993-5 (1992). The cell line recommended by ISO is a preferred established cell line obtained from recognized repositories as American Type Culture Collection (ATCC). Chinese hamster ovary cells (ATCC CHO K1) were used in this investigation.

\section{Results and discussion}

Four different membranes with different compositions were synthesized in accordance to the stoichiometry presented in Table 1. The M1 sample is the ungrafted chitosan membrane, which corresponds to the control. M2 and M3 samples represent chitosan membranes grafted with HEMA and AA, respectively. To prepare M6 sample the vinyl monomers were used together in the graft reactions at 1:1 molar ratio. The graft copolymerization is a random and heterogeneous process (Fig. 1). Side chains could be covalently linked to the $\mathrm{C}-2$ free amino groups in deacetylated units or to the hydroxyl groups in the C-3 and C6 carbons either of acetylated or deacetylated units (Lloyd et al., 1998; Berger et al., 2004). They are formed by the arrangement 


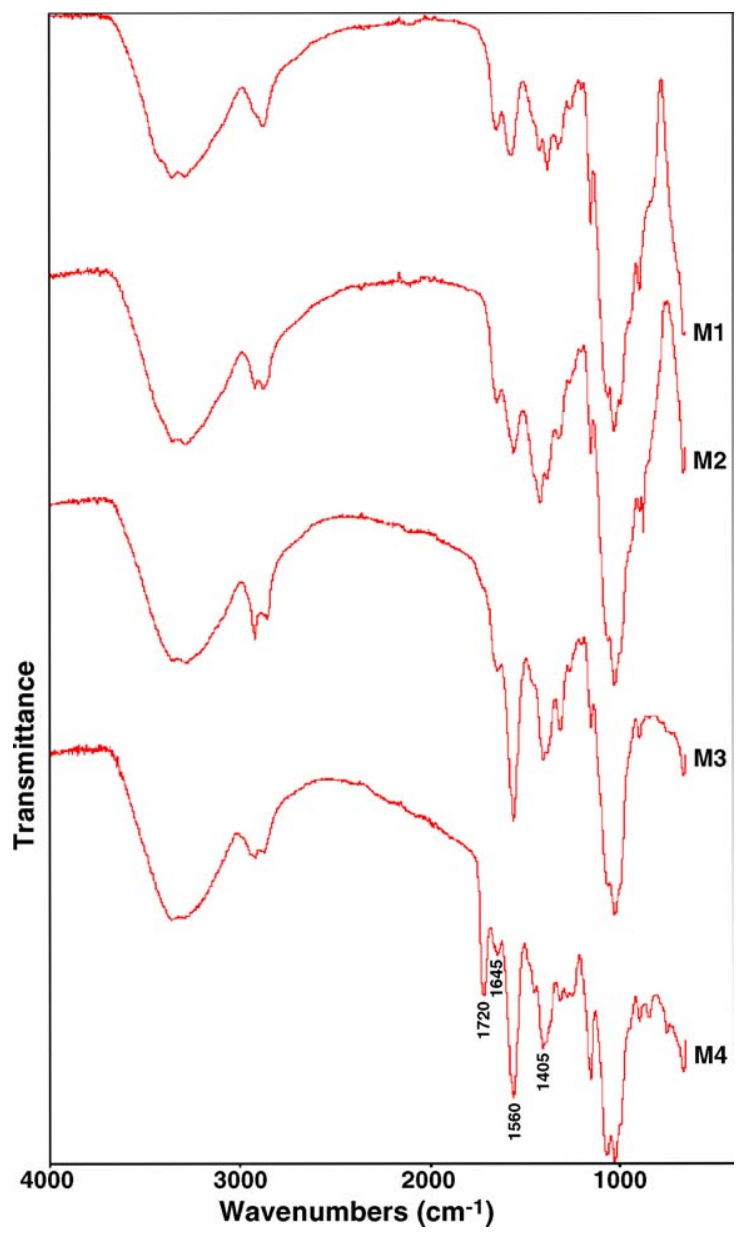

Fig. 2. FTIR aligned spectrums of M1, M2, M3 and M4 samples.

of the HEMA and AA monomers either in blocks or randomly. Despite these variables, the success of graft copolymerization reactions was confirmed by FTIR and DMTA.

Fig. 2 represents the FTIR aligned spectra of all samples. The absorption at $1720 \mathrm{~cm}^{-1}$ is attributed to the $\mathrm{C}=\mathrm{O}$ asymmetric stretching of the ester of poly(HEMA), confirming that M2 and $\mathbf{M} 4$ samples were grafted by this polymer. The very intense characteristic band at $1560 \mathrm{~cm}^{-1}$ is due to the $\mathrm{C}(\mathrm{O}$ asymmetric stretching of the carboxylate anion from poly(AA) grafted onto M3 and M4 chitosan membranes. This is reconfirmed by other sharp peak at $1405 \mathrm{~cm}^{-1}$, which is related to the symmetric stretching mode of the carboxylate anion (Hu et al., 2002; Mahdavinia et al., 2004). The salt form of the free carboxylic group is expected since the membranes were neutralized with sodium hydroxide solution to remove them from the plates. To be sure there were not residual monomers on membranes, the final extraction solvent was analysed by UV spectroscopy (data not shown). No signal was obtained, showing that all the monomers were already extracted.

The DMTA represents a powerful technique to determine the miscibility of different compounds, which is an important help to characterize the graft copolymers based on chitosan, prepared in this work. We can normally consider that the detection of a single peak in $\tan \delta$ is a sufficient criterion to assume the mis-

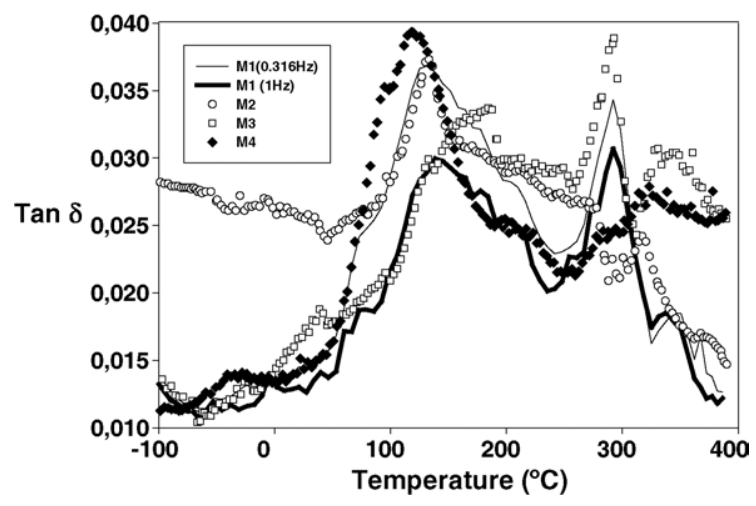

Fig. 3. DMTA aligned curves of M1, M2, M3 and M4 samples.

cibility, when compounds do not have $T_{\mathrm{g}}$ values too close. It is important to elucidate whether the polymer blend is miscible or immiscible, since it plays an important role in the composite behaviour (Wu et al., 2004).

Fig. 3 represents the DMTA aligned curves of all samples. The membrane M1 was prepared to obtain the thermal characterization of the chitosan used in this work. For that reason and in order to obtain additional information about the nature of the movements involved, the DMTA of this membrane was recorded at two different frequencies. The results obtained at $1 \mathrm{~Hz}$ showed five important peaks at $0,72,131,201$ and at $292^{\circ} \mathrm{C}$. The first peak at $0{ }^{\circ} \mathrm{C}$ is related with the chain movements induced by the water presence. The peak at $72^{\circ} \mathrm{C}$ can be attributed to some water induced relaxation due to the chitosan hydrophylicity. This transition can be detected also by DSC for a 5-25\% range of moisture, due to the enthalphic association between water and the carbohydrate. The second transition at $131^{\circ} \mathrm{C}$ (Wu et al., 2004; Mucha and Pawlak, 2005) is normally ascribed to the glass transition temperature. Concerning the peak at $200{ }^{\circ} \mathrm{C}$ it is difficult to recognize it as a consequence of any molecular movement. Dong and co-workers have reported that the $T_{\mathrm{g}}$ of chitosan is around $200^{\circ} \mathrm{C}$, which is very close to the degradation temperature onset. One possible justification for the peak at $200{ }^{\circ} \mathrm{C}$ is some liquid-liquid transition, which corresponds to the motion of very long segments or even the whole chain (Dong et al., 2004). According to the results obtained at two different frequencies $(0.316$ and $1 \mathrm{~Hz})$ for M1 the only peak that showed to be frequency dependent was that recognized as the $T_{\mathrm{g}}\left(131^{\circ} \mathrm{C}\right)$, as expected since it represents an alfa-relaxation. For that reason, it is assumed that the $T_{\mathrm{g}}$ of the chitosan used in this work is $131^{\circ} \mathrm{C}$. The final peak at $300^{\circ} \mathrm{C}$ is widely recognized as related to the decomposition temperature (Jiang et al., 1996; Xiao et al., 2003). It starts generally around $230^{\circ} \mathrm{C}$, and can also be confirmed by using the thermogravimetric data published (Liu et al., 2003). The initial temperature for decomposition can also be reported (slight different values) to the dependence of the acetyl content and the degree of polymerisation, which influences thermal stability. A small peak at $350{ }^{\circ} \mathrm{C}$ was also detected, and most probably is due to the main chain cleavage.

The M2 curve shows a single and sharp peak in the alfatransition region. This observation allows us to consider a perfect miscibility between the poly(HEMA) and chitosan. The peak 
that corresponds to the degradation has shifted to higher temperatures, which indicates increasing in thermostability. It should be also referred that the peak around $75^{\circ} \mathrm{C}$ disappeared, indicating a different water behaviour in chitosan when poly(HEMA) is grafted. Another important characteristic of the copolymer prepared is the lower intensity of the peaks at 0 and $72{ }^{\circ} \mathrm{C}$. As mentioned before, they are related with water induction movements. This fact shows a decrease in the capacity to retain water due to the introduction of the poly(HEMA). The M3 curve shows that the graft copolymer with AA has a higher $T_{\mathrm{g}}$ when compared with the chitosan (M1). This observation is related most probably with the reaction between amino groups of chitosan and carboxylic groups of poly(AA) from adjacent chains, resulting in the formation of a stiffer material. The degradation temperature onset was also shifted to higher temperature showing an improvement in the chitosan thermal properties. A careful examination of the behaviour depicted in the degradation curve shows three regions: a small peak in the region of $240-250{ }^{\circ} \mathrm{C}$, due to water loss caused by the amide formation on the carboxylate group in the poly(AA); a second peak between 250 and $320^{\circ} \mathrm{C}$ due to the deacytelation of chitosan and decarboxylation of poly(AA) main chains, and the last peak after $320^{\circ} \mathrm{C}$ mainly due to chain cleavage of chitosan. In what concerns the M4 sample, the glass transiton temperature was shifted to higher temperature compared with poly(AA) and poly(HEMA) themselves, $106^{\circ} \mathrm{C}$ (Kim et al., 2003; Jovanovic et al., 2004) and $55^{\circ} \mathrm{C}$ (Ende and Peppas, 1996), respectively, and to lower temperature compared with chitosan itself, which indicates some interaction between all the components. However, a small peak in $100{ }^{\circ} \mathrm{C}$ was also detected. This effect could be due to the water presence.

The chitosan membranes containing only HEMA (M2) tend to be thicker and have more wrinkles than those composed exclusively of chitosan (M1). Chitosan membranes grafted with AA (M3) are able to absorb large contents of water, and drastically increase the volume, which makes this film very fragile for handling. Swelling properties are important to mimic the skin's moisture and to incorporate drugs in the polymeric matrix, however, too much impairs the physical stability of the membranes. Thus, it is expected that chitosan membranes grafted with both monomers are able to mix their properties to obtain membranes with ideal swelling, mechanical and physical profiles.

The results obtained in swelling studies are shown in Fig. 4. The DMTA analysis has already shown that introduction of poly(HEMA) decreases the chitosan capacity to retain water whereas poly(AA) increases the swelling properties. M3 sample, the membrane with the highest content in AA, showed the highest swelling capacity $(Q=2860 \%)$, which was about 14 -fold more water absorbent than that of the control (M1) but it is fragile to handle. Conversely, M2, which is the membrane with the highest HEMA content, presented the lowest swelling degree $(Q=220 \%)$, which was almost the same as that of the control $(Q=223 \%)$. Chitosan membrane grafted with equimolar ratio of HEMA and AA (M4) has swelling properties balanced according to the composition of each monomer. It is able to absorb five-fold more water than chitosan membrane (M1) without impairing its physical stability. The large standard deviations

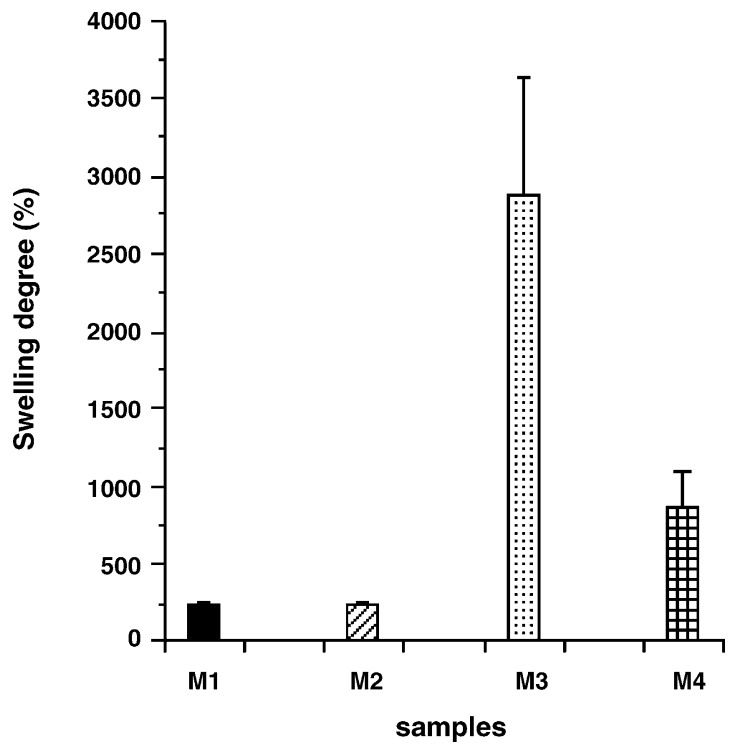

Fig. 4. Swelling degrees $(Q)$ of membranes.

measured in some triplicates reflect the heterogeneity of graft reactions. Depending on the membrane portion taken for the test, the results could differ drastically between themselves due to the random insertion of the vinyl side chain grafted onto chitosan. This heterogeneous behaviour was also observed in attempts to determine the hydrophilic character of membrane surfaces by measurement of the contact angle through pendent water drop method (data not shown). In so far as films were screened, the contact angle measurements differed drastically between themselves from one point to another, reinforcing the idea of random graft copolymerization of HEMA and AA onto chitosan.

Since these membranes were designed to be used topically in wound healing, it is important to evaluate its tissue and blood compatibility. Furthermore, the thrombogenic character is a desirable property in membranes. Once the protein adhesion constitutes the first step to initiate the coagulation cascade, membranes can accelerate the thrombus formation, stopping haemorrhage and helping in the healing process.

Fig. 5 shows the weights of the blood clots obtained on thrombogenicity test. It was observed that clot formation was lower in

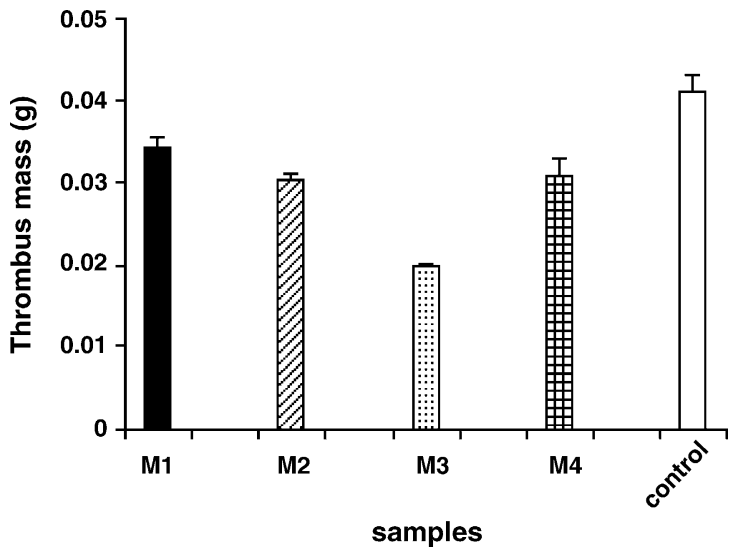

Fig. 5. Thrombogenic index obtained for the membranes. 
the membranes than in the control and for this reason, the polymers are classified as non-thrombogenic (Imai and Nose, 1972). This characteristic is directly related to the hydrophilicity of the materials. When placed in contact with a hydrophobic surface, proteins adsorb to it in a strong and irreversible way, while at hydrophilic surfaces proteins adsorb weakly and reversibly (Changsheng et al., 2003).

This relation between hydrophilicity and thrombosis was confirmed by the lower value of thrombus weight that was formed when blood contacted with M3, the most hydrophilic of all membranes. However, it could be noticed that M4 was almost so "thrombogenic" as M2, despite its content in AA. This result shows that it is possible to obtain a chitosan-based membrane with some hydrophilic character, but without losing its thrombogenic properties, by mixing HEMA and AA on graft copolymerization of chitosan.

Fig. 6 represents values of haemolysis obtained for all membranes. The method is based on measurement of the haemoglobin released by haemolysis. Haemolysis is regarded as an especially significant screening test, once it provides quantification of small levels of plasma haemoglobin, which may not be measurable under in vivo conditions. As reported in literature (ISO 10993-4 (1999)), it is not possible to define a universal level of acceptable or unacceptable amounts of haemolysis. Although by definition a blood-compatible material should be non-haemolytic, in practice several medical devices cause haemolysis. This means that when such haemolytic effect takes place, it is important to make sure that clinical benefits overcome the risks and that the values of haemolysis are within acceptable limits.

The ungrafted chitosan sample (M1) follows into the slightly haemolytic category according to ASTM F 756-00 (2000) while M2, which represents chitosan-graft-HEMA sample can be considered non-haemolytic. This might be due to the change in surface composition because the blocking of reactive free amino groups from chitosan by grafting poly(HEMA) at this moieties. On the other hand, $\mathbf{M} 3$ presents a haemolytic character. It could be noticed that the mixture between AA and HEMA to graft

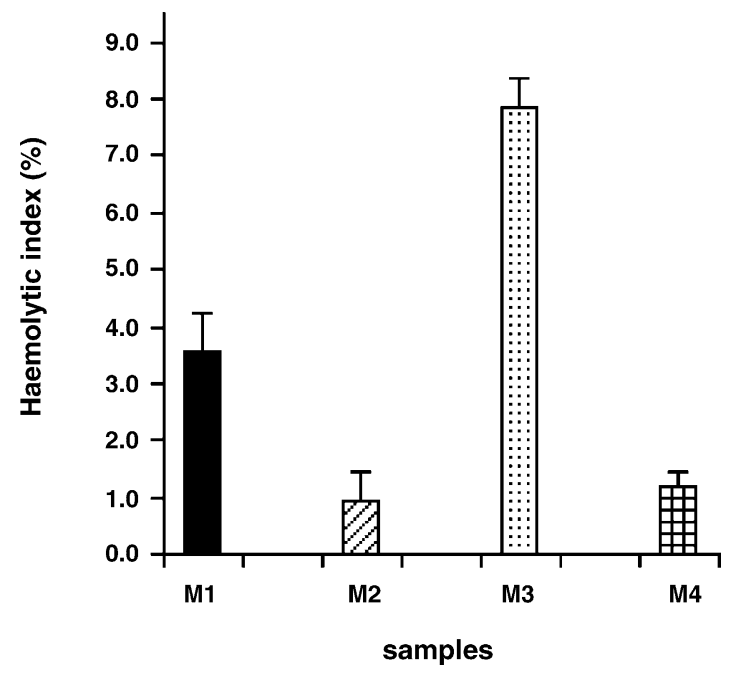

Fig. 6. Haemolytic index obtained for the membranes. chitosan (M4) generates membrane almost non-haemolytic as is $\mathbf{M} 2$ and it has eight-fold improved the haemolytic behaviour if compared with chitosan grafted just by AA (M3). Over again, it is clear that the mixture of HEMA and AA in graft copolymerization of chitosan generates membrane with better properties than those mono-vinyl-grafted.

Determination of cytotoxicity can be either qualitative or quantitative (ISO 10993-5, 1992). The qualitative evaluation examines cells microscopically to assess for changes in general morphology, vacuolization, detachment and cell and membrane lysis. Quantitative evaluation of cell death, inhibition of cell growth, cell proliferation or colony formation (Rogero et al., 2000). In this study, determination of cytotoxicity was performed by quantitative evaluation of cell viability.

The method is based on the procedure originally developed by Borefreund and Puerner (1984) for the screening of cytotoxic agents, in general, over monolayer cells. This method is based on the quantitative assessment of surviving viable cells following exposure to the toxic agent, by incubation with the supravital dye tetrazolium compound MTS and an electron coupling reagent PMS. MTS is bioreducted by viable cells into a formazan product that is soluble in tissue culture medium and a colorimetric analysis of the incorporate dye is performed. The amount of MTS, the marker of cell viability, taken up by the cell population, is directly proportional to the number of viable cells in the culture.

Fig. 7 shows the results of the cytotoxicity test, which can predict the biocompatibility of the samples. Comparing membranes with the negative control (HDPE), M1 and M2 could be considered harmless at the cytotoxic point of view. M3 sample showed a cytotoxic effect over the cells since it caused $60 \%$ of cell death. However, after grafting chitosan with AA and HEMA (M4), the cell viability was almost two-fold improved in comparison with the membrane grafted only by AA (M3). As well as shown on further assays, the cell viability results indicate that the introduction of some HEMA content in chitosan-graft-AA is important to improve also this property.

In conclusion, all membranes with AA in their composition are able to swell, but M4, which are grafted by equimolar ratio of HEMA and AA, keeps good swelling capacity without compromising its physical stability. Besides, M4 had improved

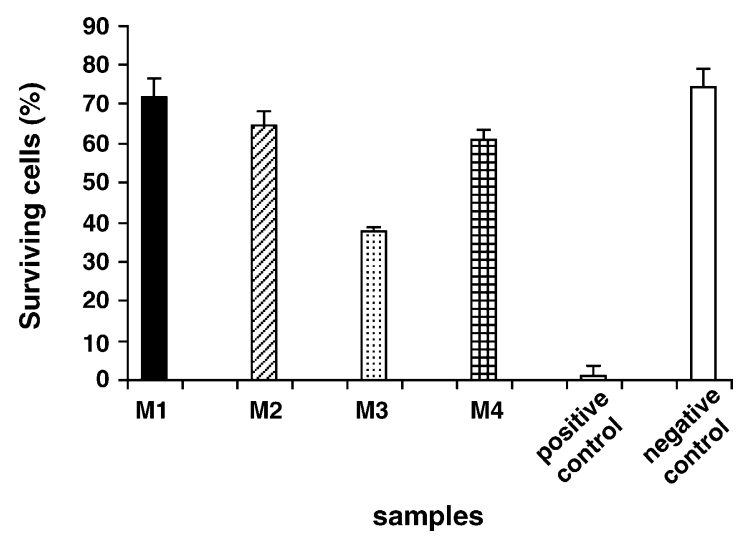

Fig. 7. Citotoxicity test of the membranes. 
thrombogenic, non-haemolytic and cytotoxic profiles in comparison with $\mathbf{M} 3$ because its content in HEMA. All those advantages make M4 a suitable biomaterial to be applied as a topical drug delivery system on wound healing. This work showed that is possible to obtain chitosan membranes grafted both by AA and HEMA with improved properties than chitosan grafted just by either AA or HEMA.

\section{Acknowledgements}

We thank the National Council of Technological and Scientific Development $(\mathrm{CNPq})$, the Co-ordination for Improvement of Superior Level Students (CAPES), for scholarship to K.S.C.R. Santos, and the University of Coimbra (UC) for financial support.

\section{References}

American Society for Testing and Materials, 2000. ASTM F 756-00: Standard Practices for Assessment of Haemolytic Properties of Materials, Philadelphia.

Athawale, V.D., Lele, V., 2001. Recent trends in hydrogels based on starchgraft-acrylic acid: a review. Starch/Staerke 53, 7-13.

Berger, J., Reist, M., Mayer, J.M., Felt, O., Gurny, R., 2004. Structure and interactions in chitosan hydrogels formed by complexation or aggregation for biomedical applications. Eur. J. Pharm. Biopharm. 57, 35-52.

Borefreund, E., Puerner, J.A., 1984. A simple quantitative procedure using monolayer cultures for cytotoxicity assay (HTD/NR-90). J. Tissue Cult. Method 9, 7-9.

Carenza, M., 1992. Recent achievements in the use of radiation polymerization and grafting for biomedical applications. Radiat. Phys. Chem. 39, 485-493.

Casimiro, M.H., Botelho, M.L., Leal, J.P., Gil, M.H., 2005. Study on chemical, UV and gamma radiation-induced grafting of 2hydroxyethyl methacrylate onto chitosan. Rad. Phys. Chem. 72, 731735.

Changsheng, Z., Xiangdong, L., Motoyoshi, N., Norio, N., 2003. Blood compatible aspects of DNA-modified polysulfone membrane-protein adsorption and platelet adhesion. Biomaterials 24, 3747-3755.

Chen, T., Kumar, G., Harris, M.T., Smith, P.J., Payne, G.F., 2000. Enzymatic grafting of hexyloxyphenol onto chitosan to alter surface and reological properties. Biotechnol. Bioeng. 70, 564-573.

Chirkov, S.N., 2002. The antiviral activity of chitosan (review). Appl. Biochem. Microbiol. 38, 1-8.

Dong, Y., Ruan, Y., Wang, H., Zhao, Y., Bi, D., 2004. Studies on glass transition temperature of chitosan with four techniques. J. Appl. Polym. Sci. 93, 1553-1558.

Ende, M.T.A., Peppas, N.A., 1996. Transport of ionizable drugs and proteins in crosslinked poly(acrylic acid) and poly(acrylic acid-co-2-hydroxyethyl methacrylate) hydrogels. I. Polymer characterization. J. Appl. Polym. Sci. 59, 673-685.

Harish Prashanth, K.V., Tharanathan, R.N., 2003. Studies on graft copolymerization of chitosan with synthetic monomers. Carbohydr. Polym. 54, 343-351.

Henriksen, I., Green, K.L., Smart, J.D., Smistad, G., Karlsen, J., 1996. Bioadhesion of hydrated chitosans: an in vitro and in vivo study. Int. J. Pharm. $145,231-240$.

Hu, Y., Jiang, X., Ding, Y., Ge, H., Yuan, Y., Yang, G., 2002. Synthesis and characterization of chitosan-poly(acrylic acid) nanoparticles. Biomaterials 23, 3139-3201.

Illum, R., 1998. Chitosan and its use as a pharmaceutical excipient. Pharm. Res. 15, 1326-1331.

Imai, Y., Nose, Y.J., 1972. New method for evaluation of antithrombogenicity of materials. Biomed. Mater. Res. 6, 165-172.
International Standard Organization, 1992. ISO 10993-5: Biological Evaluation of Medical Devices - Part 5 - Tests for Cytotoxicity: In Vitro Methods, Geneva.

International Standard Organization, 1999. ISO 10993-4: Biological Evaluation of Medical Devices - Part 4 - Selection of Tests for Interaction with Blood, Geneva.

Jiang, H., Caracci, W., Bumming, S., Cooper, T., Adams, W., 1996. Optical waveguiding and morphology of chitosan thin films. J. Appl. Polym. Sci. 61, 1163-1171.

Jovanovic, R., McKenna, T.F., Dubé, M.A., 2004. Empirical modeling of butyl acrylate/vinyl acetate/acrylic acid emulsion-based pressure-sensitive adhesives. Macromol. Mater. Eng. 289, 467-474.

Kim, S.J., Lee, K.J., Lee, S.M., Kim, I.Y., Lee, Y.M., Kim, S.I., 2003. Thermal characteristics of IPNs compsed of poly(propylene glycol) and poly(acrylic acid). J. Appl. Polym. Sci. 88, 2570-2574.

Kurika, K., 1996. Chitin and chitosan graft copolymers. In: Salomone, J.C. (Ed.), Polymeric Materials Encyclopedia, vol. 2. CRC Press, New York, pp. 1205-1208.

Lee, J.W., Kim, S.Y., Kim, S.S., Lee, Y.M., Lee, K.H., Kim, S.J., 1999. Synthesis and characteristics of interpenetrating polymer network hydrogel composed of chitosan and poly(acrylic acid). J. Appl. Polym. Sci. 73, $113-120$.

Lee, J.S., Kumar, R.N., Rozman, H.D., Azemi, B.M.N., 2005. Pasting, swelling and solubility properties of UV initiated starch-graft-poly(AA). Food Chem. 91, 203-211.

Li, Q., Dunn, E.T., Grandmaison, E.W., Goosen, M.F.A., 1997. Applications and properties of chitosan. In: Goosen, M.F.A. (Ed.), Applications of Chitin and Chitosan. Technomic Publishing Company, Lancaster, pp. 3-29.

Lim, S.H., Hudson, S.M., 2003. Review of chitosan and its derivatives as antimicrobial agents and their uses as textile chemicals. J. Macromol. Sci.: Polym. Rev. C43, 223-269.

Liu, Y., Liu, Z., Zhang, Y., Dung, K., 2003. Graft copolymerization of methyl acrylate onto chitosan initiated by potassium diperodatocuprate (III). J. Appl. Polym. Sci. 89, 2283-2289.

Lloyd, L.L., Kennedy, J.F., Methacanon, P., Paterson, M., Knill, C.J., 1998 Carbohydrate polymers as wound management aids. Carbohydr. Polym. 37, 315-322.

Mahdavinia, G.R., Pourjavadi, A., Hosseinzadeh, H., Zohuriaan, M.J., 2004. Modified chitosan 4. Superabsorbent hydrogels from poly(acrylic acid-coacrylamide) grafted chitosan with salt- and $\mathrm{pH}$-responsiveness properties. Eur. Polym. J. 40, 1399-1407.

Mucha, M., Pawlak, A., 2005. Thermal analysis of chitosan and its blends. Thermochim. Acta 427, 69-76.

Muzzarelli, R.A.A., 1991. Role of lysozyme and $N$-acetil- $\beta$-Dglucosaminidase in the resorption of wound dressings. In: Brine, C.J., Sandford, P.A., Zikakis, J.P. (Eds.), Chitin and Chitosan: Sources, Chemistry, Biochemistry, Physical Properties and Applications. Elsevier Applied Science, New York, pp. 25-33.

Muzzarelli, R.A.A., 1997. Human enzymatic activities related to the therapeutic administration of chitin derivatives. Cell. Mol. Life Sci. 53, 131140.

Okamoto, Y., Yano, R., Miyatake, K., Tomohiro, I., Shigemasa, Y., Minami, S., 2003. Effects of chitin and chitosan on blood coagulation. Carbohydr. Polym. 53, 337-342.

Olsen, R., Schwartzmiller, D., Weppner, W., Winandy, R., 1988. Biomedical applications of chitin and its derivatives. In: Skjåk-Bræk, G., Anthonsen, T., Sandford, P. (Eds.), Chitin and Chitosan: Sources, Chemistry, Biochemistry, Physical Properties and Applications. Elsevier Applied Science, London, pp. 813-828.

Rabea, E.I., Badawy, M.E.T., Stevens, C.V., Smagghe, G., Steurbaut, W., 2003. Chitosan as antimicrobial agent: applications and mode of action. Biomacromolecules 4, 1457-1465.

Rao, S.B., Sharma, C.P., 1997. Use of chitosan as biomaterial: studies on its safety and haemostatic potential. J. Biomed. Mater. Res., Part A 34, 21-28.

Ravi Kumar, M.N.V., 2000. A review of chitin and chitosan applications. React. Funct. Polym. 46, 1-27. 
Rogero, S.O., Higa, O.Z., Saiki, M., Correa, O.V., Costa, I., 2000. Cytotoxicity due to corrosion of ear piercing studs. Toxicol. In Vitro 14, 497-504.

Sashiwa, H., Aiba, S.-I., 2004. Chemically modified chitin and chitosan as biomaterials. Prog. Polym. Sci. 29, 887-908.

Singh, D.K., Ray, A.R., 1994. Graft copolymerization of 2-hydroxyethylmethacrylate onto chitosan films and their blood compatibility. J. Appl. Polym. Sci. 53, 1115-1121.

Singla, A.K., Chawla, M., 2001. Chitosan: some pharmaceutical and biological aspects—an update. J. Pharm. Pharmacol. 53, 1047-1067.

US Pharmacopeia XXIII, 1994. US Pharmacopeial Convention, Rockville, MD, p. 119.
Wu, Y.-B., Yu, S.-H., Mi, F.-L., Wu, C.-W., Shyu, S.-S., Peng, C.-K., Chao, A.-C., 2004. Preparation and characterization on mechanical and antibacterial properties of chitosan/cellulose blends. Carbohydr. Polym. 57, 435-440.

Xiao, C., Zhang, J., Zhang, Z., Zhang, L., 2003. Study of blend films from chitosan and hydroxypropyl guar gum. J. Appl. Polym. Sci. 90, 1991-1995.

Zheng, L.-Y., Zhu, J.-F., 2003. Study on antimicrobial activity of chitosan with different molecular weights. Carbohydr. Polym. 54, 527530. 